\title{
ARTICLE
}

Received 5 Dec 2012 | Accepted 4 Mar 2013 | Published 9 Apr $2013 \quad$ DOl: 10.1038/ncomms2691

\section{Electrically driven polarized single-photon emission from an InGaN quantum dot in a GaN nanowire}

\author{
Saniya Deshpande ${ }^{1}$, Junseok Heo ${ }^{1, \dagger}$, Ayan Das $^{1} \&$ Pallab Bhattacharya ${ }^{1}$
}

In a classical light source, such as a laser, the photon number follows a Poissonian distribution. For quantum information processing and metrology applications, a non-classical emitter of single photons is required. A single quantum dot is an ideal source of single photons and such single-photon sources in the visible spectral range have been demonstrated with III-nitride and II-VI-based single quantum dots. It has been suggested that shortwavelength blue single-photon emitters would be useful for free-space quantum cryptography, with the availability of high-speed single-photon detectors in this spectral region. Here we demonstrate blue single-photon emission with electrical injection from an $\ln _{0.25} \mathrm{Ga}_{0.75} \mathrm{~N}$ quantum dot in a single nanowire. The emitted single photons are linearly polarized along the $c$ axis of the nanowire with a degree of linear polarization of $\sim 70 \%$.

\footnotetext{
${ }^{1}$ Department of Electrical Engineering and Computer Science, Center for Photonics and Multiscale Nanomaterials, University of Michigan, 1301, Beal Avenue, Ann Arbor, Michigan 48109-2122, USA. †Present address: Department of Electrical and Computer Engineering, Ajou University, Suwon 443-749, South Korea. Correspondence and requests for materials should be addressed to P.B. (email: pkb@eecs.umich.edu).
} 
S ince the early demonstration of a quantum dot (QD) singlephoton source $e^{1-5}$, there have been numerous reports on single-photon emission in a wide range of temperatures, with or without the use of a microcavity and with optical or electrical excitation ${ }^{1-20}$. Generally, the electrically driven singlephoton emitters are all-epitaxial heterostructures. However, polarization control is not easily achieved. For quantum cryptography protocols based on polarization encoding, it is desirable to have a single-photon source whose output is not randomly polarized, but has a fixed polarization depending on the size and shape of the emitter ${ }^{21,22}$.

$\mathrm{GaN}$ and $\mathrm{In}(\mathrm{Ga}) \mathrm{N}$ nanowires can be epitaxially grown catalyst free on silicon substrates with aerial density in the range of $10^{8}-10^{11} \mathrm{~cm}^{-2}$ and lengths up to a few micrometres ${ }^{23-32}$. The nanowires grow vertically in the wurtzite crystalline form and optical emission from them in the spectral range of $366-700 \mathrm{~nm}$ has been recorded by varying the In composition. Extensive structural characterization indicates that the nanowires are relatively free of extended defects ${ }^{23,24,27-29,31-34}$. They can be doped $\mathrm{p}$ - and n-type with suitable dopant species up to levels similar to those achieved in planar structures. The surface recombination velocity in $\mathrm{GaN}$ nanowires is $\sim 10^{3} \mathrm{~cm} \mathrm{~s}^{-1}$, two orders of magnitude smaller than that on a planar surface of $\mathrm{GaAs}$ (ref. 35). It is possible to grow one or multiple $\operatorname{In}(\mathrm{Ga}) \mathrm{N}$ disks in $\mathrm{Al}(\mathrm{Ga}) \mathrm{N}$ nanowires to provide quantum confinement, such as in a quantum well ${ }^{36-38}$. However, due to the radial strain relaxation during growth, the piezoelectric polarization field in the disks can be much smaller than in equivalent quantum wells. If the diameter of the nanowire is made small enough and the thickness of the disk is $\sim 2-3 \mathrm{~nm}$, then quasi-three-dimensional confinement, or a $\mathrm{QD}$, is realized. In this bottom-up growth approach, the size of the nanowire and therefore the size of the QD can be carefully controlled.

In this work we have investigated single-photon emission from a single $\mathrm{In}_{0.25} \mathrm{Ga}_{0.75} \mathrm{~N}$ QD in a single $\mathrm{GaN}$ nanowire with electrical injection. The $\mathrm{GaN}$ nanowire contains a $\mathrm{p}-\mathrm{n}$ junction, and the injected electrons and holes recombine in the $\mathrm{In}_{0.25} \mathrm{Ga}_{0.75} \mathrm{~N}$ QD. The InGaN/GaN dot-in-nanowire heterostructure was grown by plasma-assisted molecular beam epitaxy (PA-MBE) on silicon. The single dot/nanowire junction diode is fabricated on (001) silicon and the experiments have been performed at an ambient temperature of $10 \mathrm{~K}$. The junction temperature is estimated to be higher due to Joule heating, as discussed later. Sharp exciton and biexciton transitions are observed at $\lambda=436.56 \mathrm{~nm}$ and $435.03 \mathrm{~nm}$, respectively, in the electroluminescence spectra. Single-photon emission is observed at the biexciton emission wavelength with a value of the secondorder correlation function $g^{(2)}(0)$ equal to 0.25 . The emitted photons are linearly polarized along the $c$ axis of the nanowire. The radiative lifetime of biexciton emission obtained from timeresolved photoluminescence (TRPL) measurements is $711 \mathrm{ps}$, and this value is in excellent agreement with the value of the lifetime obtained from analysis of second-order correlation data. Singlephoton emission with a larger recombination lifetime of $1.1 \mathrm{~ns}$ was also observed at the exciton energy. The value of $g^{(2)}(0)$ for the exciton transition is 0.16 .

\section{Results}

Epitaxial growth and structural characterization. The device used to demonstrate single-photon emission in this work was a $\mathrm{GaN}$ nanowire $\mathrm{p}-\mathrm{n}$ junction with an $\mathrm{InGaN}$ dot in the active region. The InGaN/GaN dot-in-nanowire heterostructure, shown schematically in Fig. 1a for a single nanowire, was grown on (111) Si substrate by PA-MBE. Catalyst-free growth of the nanowire sample was carried out under nitrogen-rich conditions (see
Methods). Each individual nanowire contains a single InGaN QD, surrounded by undoped GaN barriers, grown on top of Si-doped $\mathrm{GaN}$ (n-GaN). At this stage, the nanowire (and QD) diameter is $\sim 25 \mathrm{~nm}$. Following the growth of the QD and GaN barrier, $\mathrm{Mg}$ doped $\mathrm{GaN}$ (p-GaN) was grown on top at a lower substrate temperature. The grown sample was structurally characterized by scanning electron microscope (SEM) imaging, seen in Fig. 1b, which yields a nanowire density of $\sim 10^{11} \mathrm{~cm}^{-2}$. Figure $1 \mathrm{c}$ shows the transmission electron microscope (TEM) image of a single nanowire diode. The structure of the InGaN QD was studied by scanning TEM (STEM). As seen in the high-resolution image in the inset of Fig. 1c, both InGaN and GaN regions have perfect crystalline structure. The InGaN/GaN interface appears to be smooth and free of stacking faults. STEM imaging performed on several single dot-in-nanowire samples reveals that the QD diameter and thickness are $25 \pm 5 \mathrm{~nm}$ and $\sim 2 \mathrm{~nm}$, respectively. Energy-dispersive X-ray (EDX) spectroscopy was performed to confirm the location, composition and thickness of the QD region. An EDX line scan with a $0.2-\mathrm{nm}$ resolution was performed along the nanowire growth $c$ axis (indicated by the arrow) and the results are shown in Fig. 1d. An average alloy composition of $\mathrm{In}_{0.25} \mathrm{Ga}_{0.75} \mathrm{~N}$ in the QD is derived. Additionally, we have grown InGaN nanowires of the same composition as the InGaN dots in the experimental samples and under identical growth conditions. EDX measurements performed on the nanowire ensemble yield an alloy composition of $\operatorname{In}_{0.23} \mathrm{Ga}_{0.77} \mathrm{~N}$, confirming the result of the EDX measurement on the single dot-in-nanowire (see Supplementary Fig. S1).

PL and electroluminescence measurements. Temperaturedependent PL spectra from an identical QD-nanowire ensemble sample, without the $\mathrm{p}-\mathrm{GaN}$ grown on top, are presented in Fig. 2a. Strong InGaN QD emission was observed at a wavelength of $\sim 450 \mathrm{~nm}$, with a linewidth of $\sim 14 \mathrm{~nm}$, which is similar to that reported for planar InGaN/GaN single quantum wells ${ }^{39}$. As nanowires are non-resonantly excited, weak emission from GaN is also seen at $\sim 358 \mathrm{~nm}$. The blue shift of the $\mathrm{GaN}$ emission compared with the $365-\mathrm{nm}$ emission of bulk $\mathrm{GaN}$ is a signature of quantum confinement in the nanowires ${ }^{40}$. The temperature variation of the InGaN QD luminescence peak is plotted in Fig. $2 \mathrm{~b}$ and the trend is analysed with the Varshni equation ${ }^{41}$. The S-type behaviour is not seen, indicating the absence of any significant compositional inhomogeneity or clustering in the InGaN region ${ }^{42-44}$. A radiative efficiency $\eta_{\mathrm{r}}$ of $52 \%$ is estimated from the temperature-dependent data, assuming that the nonradiative recombination centres are frozen at $10 \mathrm{~K}$ (see Supplementary Fig. S2).

Nanowires with $\mathrm{p}-\mathrm{n}$ junctions were removed from the as-grown substrate and transferred to an oxide-coated silicon wafer, to artificially obtain a nanowire density of $\sim 10^{8} \mathrm{~cm}^{-2}$. Single nanowires chosen after SEM imaging were contacted using electron beam lithography and electron beam evaporation techniques (see Methods). Micro-PL from a single dot-innanowire measured at $10 \mathrm{~K}$, seen in Fig. 2c, exhibits sharp transitions in the blue spectral range, originating from the InGaN QD. Excitation power-dependent measurements confirm these peaks originate from the exciton and biexciton transitions.

Measured room temperature current-voltage characteristics of the single QD-nanowire $\mathrm{p}-\mathrm{n}$ junction (seen in Fig. 3a) are presented in Fig. $3 b$. The series resistance of the diode was carefully measured at room temperature (see Supplementary Fig. S3) and a value of $2.38 \mathrm{G} \Omega$ is derived in the bias range of $2-20 \mathrm{~V}$, in which single-photon measurements have been made. The diode has a very small reverse current $(\sim 1 \mathrm{pA})$ and exhibits no breakdown up to a reverse bias of $20 \mathrm{~V}$. We have measured 
a

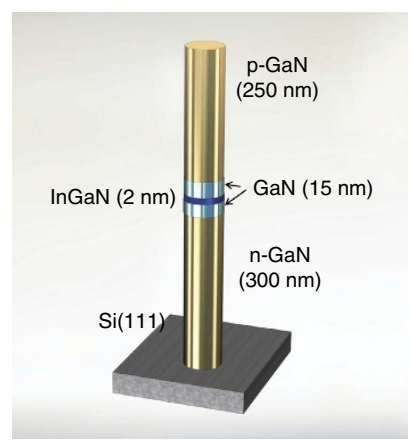

c

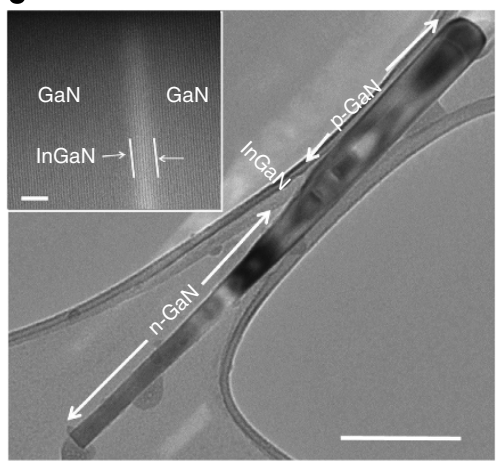

b

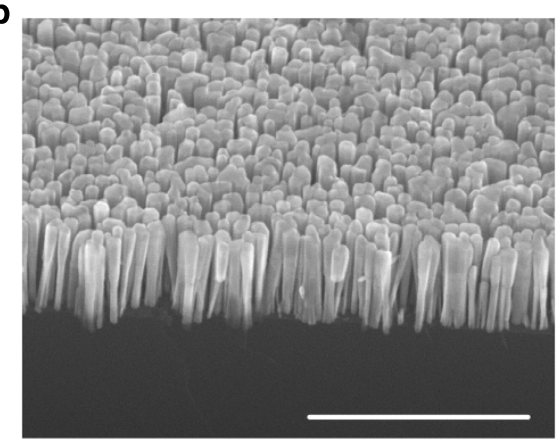

d

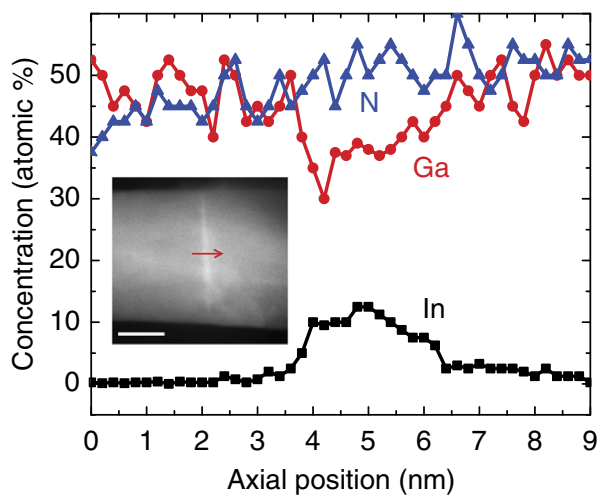

Figure 1 | Structural characterization of InGaN dot-in-nanowire p-n junction. (a) Schematic of an InGaN dot-in-GaN nanowire p-n junction grown on (111) silicon by molecular beam epitaxy. The 2-nm quantum dot is placed at the centre of doped GaN regions. (b) Scanning electron micrograph of the cleaved edge of an as-grown nanowire sample imaged at a tilt of $45^{\circ}$. The nanowire density is $\sim 10^{11} \mathrm{~cm}^{-2}$. The scale bar denotes $1 \mu \mathrm{m}$. (c) Transmission electron micrograph of a single nanowire. The scale bar denotes $100 \mathrm{~nm}$. The lower growth temperature during $\mathrm{p}$-GaN growth leads to an increase in lateral growth rate, and consequently the nanowire diameter increases. The diameter near the centre of the nanowire $p-n$ junction, where the quantum dot is located, is $25 \mathrm{~nm}$. The inset shows a high-resolution image of the $\ln _{0.25} \mathrm{Ga}_{0.75} \mathrm{~N}$ quantum dot region. The scale bar in the inset is $2 \mathrm{~nm}$. (d) EDX measurement showing the axial profile of elemental composition. Inset shows high-angle annular dark field image of the $\mathrm{GaN}_{\mathrm{I}} \mathrm{In}_{0.25} \mathrm{Ga} \mathrm{a}_{0.75} \mathrm{~N} / \mathrm{GaN}$ heterostructure. The arrow indicates the path of the EDX line scan. The width of the In peak is 2-nm full-width at half maxima. Scale bar: $10 \mathrm{~nm}$.
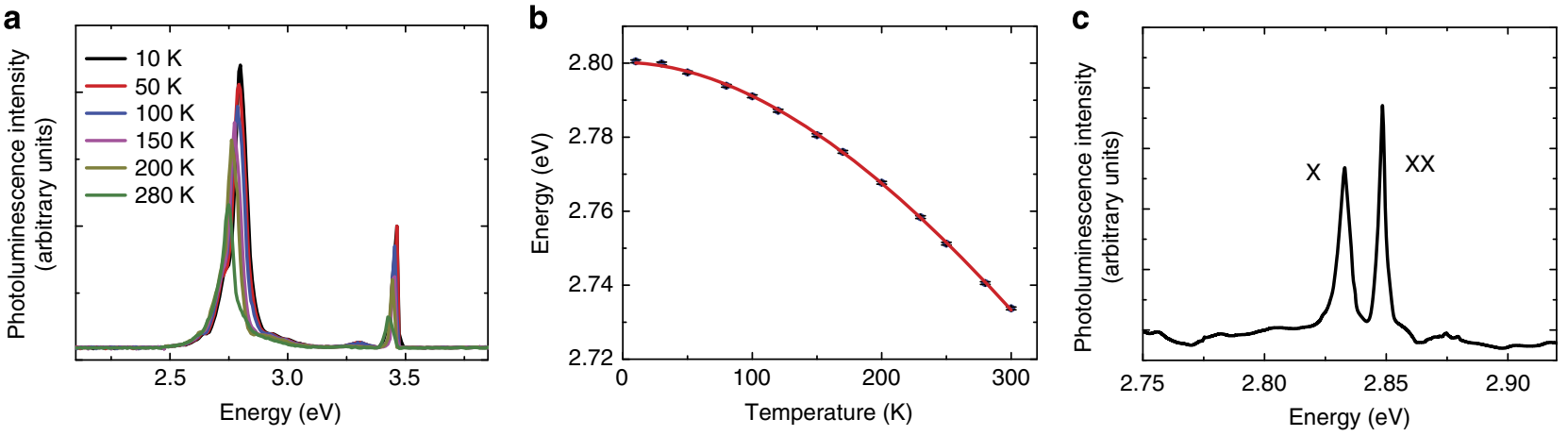

Figure 2 | InGaN quantum dot photoluminescence. (a) Temperature-dependent photoluminescence spectra from the as-grown nanowire ensemble shown in Fig. 1b. The integrated intensity of the quantum dot emission at room temperature is about half of that at $10 \mathrm{~K}$. (b) Peak energy of quantum dot emission from Fig. 2a plotted as a function of temperature with error bars representing the uncertainty due to the finite resolution of the measurement system. The solid line is the calculated variation using the Varshni equation. The Varshni parameters used for the fit are $\alpha=(8.3 \pm 0.4) \times 10^{-4} \mathrm{eV} \mathrm{K}-1$ and $\beta=825 \pm 16.7 \mathrm{~K}$. (c) Micro-photoluminescence from a single $\ln _{0.25} \mathrm{Ga}_{0.75} \mathrm{~N} / \mathrm{GaN} \mathrm{QD}$ in nanowire at $10 \mathrm{~K}$, obtained from a sample with dispersed nanowires. The excitation power was $400 \mu \mathrm{W}$.

several single nanowire $\mathrm{p}-\mathrm{n}$ junction diodes and their characteristics are similar. Typical electroluminescence (EL) spectra from the nanowire-QD device under forward bias at different injection currents are shown in Fig. 3c. Sharp resonances with linewidths ranging from 2 to $3 \mathrm{meV}$ are observed in the spectra. At low injection currents, a sharp peak at $2.84 \mathrm{eV}$ is observed. The integrated intensity of the peak increases linearly with the injection current $\left(\propto I^{1.04 \pm 0.02}\right)$. With increase in injection current, a second peak is observed at $\sim 2.85 \mathrm{eV}$, which increases quadratically with injection current $\left(\propto I^{1.93 \pm 0.09}\right)$. The linear and quadratic dependence of intensity on injected current and their subsequent saturation, as seen in Fig. 3d, confirm that the resonances are from single exciton and biexciton transitions, respectively. The measured negative biexciton binding energy of $10 \mathrm{meV}$ is in the range of previously reported values for single InGaN QDs ${ }^{45}$. 
a

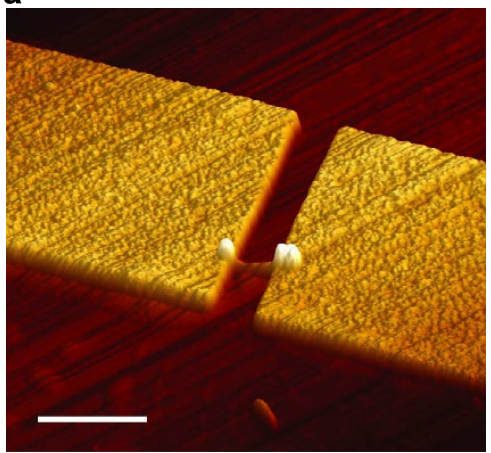

C

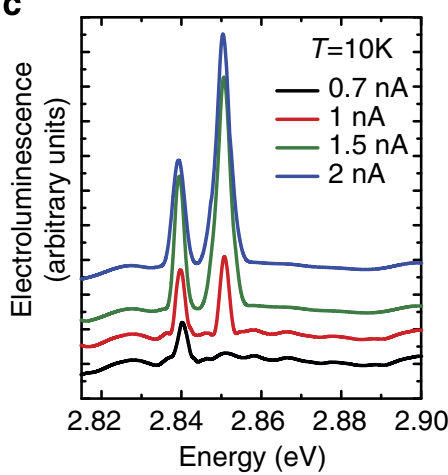

b

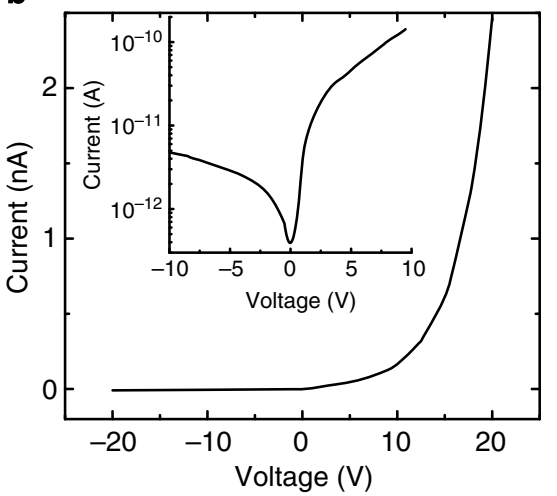

d

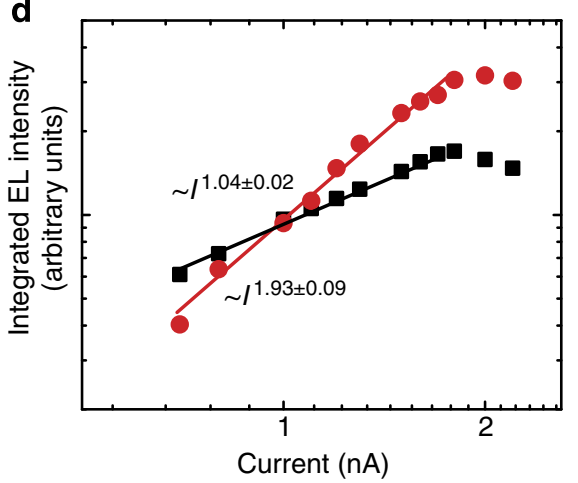

Figure 3 | Electroluminescence from InGaN dot-in-nanowire p-n junction diode. (a) Atomic force microscope image of a single dot-in-nanowire diode on (001) $\mathrm{Si}$ with Ti/Au ohmic contacts. The scale bar denotes $1 \mu \mathrm{m}$. The total thickness of the contacts is $50 \mathrm{~nm}$. (b) Room temperature current-voltage characteristics measured after annealing the contacts at $450^{\circ} \mathrm{C}$ in $\mathrm{N}_{2}$. (c) Electroluminescence from the dot-in-nanowire diode measured at $10 \mathrm{~K}$ for injection currents varying in the range $0.7-2.5 \mathrm{nA}$. (d) Integrated electroluminescence intensity of the exciton (squares) and biexciton (circles) transitions plotted as a function of injection current obtained from the data of $\mathbf{c}$. The calculated solid lines indicate that the intensity of the exciton transition increases linearly with injection current $\left(\propto l^{1.04} \pm 0.02\right.$ ) and that of the biexciton transition increases quadratically with injection current ( $\propto l^{1.93 \pm 0.09}$ ).

It is important to note at this point that because of Joule heating caused by the large series resistance of the nanowire diode, the real device (and hence the InGaN QD) temperature might be significantly larger than the ambient temperature of $10 \mathrm{~K}$. We have performed a simulation of the heat-transfer problem by solving the Laplace equation (see Methods). Figure 4a shows the simulated nanowire temperature distribution at steady state at a current density of $283 \mathrm{~A} \mathrm{~cm}^{-2}(I=2 \mathrm{nA})$. While the generated heat is effectively dissipated by silicon through $\mathrm{SiO}_{2}$, the finite heat conductivity of $\mathrm{SiO}_{2}$ results in a steady-state temperature as high as $60 \mathrm{~K}$ in the nanowire. The junction temperature was similarly simulated for other injection currents at an ambient temperature of $10 \mathrm{~K}$ and the results are shown in Fig. $4 \mathrm{~b}$. As the injection current increases, the calculated steadystate temperature increases quadratically as the heat generation is proportional to the square of the current. As shown in the transient profile in the inset of Fig. 4b, the temperature of the device reaches steady state within $30 \mathrm{~ns}$. Hence, in electroluminescence measurements, any transient temperature effect can be ignored and the injected currents determine the device temperature. In Fig. $4 \mathrm{c}$, we plot the ideal variation of the $\mathrm{In}_{0.25} \mathrm{Ga}_{0.75} \mathrm{~N}$ emission peak with current density, calculated from Varshni coefficients that were previously derived (Fig. 2b) and with temperatures that were estimated from injection current density (Fig. 4b). We may assume that in the temperature range of $10-60 \mathrm{~K}$, the exciton binding energy does not change significantly, and the variation of InGaN bandgap is the dominant contributor to the emission peak energy over this temperature range. Also plotted in Fig. $4 \mathrm{c}$ is the measured variation of the EL excitonic peak energy with injected current density. Within the limits of experimental error the two curves follow each other very closely; the measured energy shift is marginally smaller than the theoretically predicted value. This suggests that there is no significant blue shift of the emission with injection, which is important in the context of single-photon emission.

Time-resolved PL. We have investigated the temporal behaviour of the luminescence from the QD by time-resolved PL (TRPL) measurements at $10 \mathrm{~K}$. The TRPL was measured using a singlephoton detector following a high-resolution monochromator. The PL decay transient at the biexciton energy is shown in Fig. 5. The measured data were analysed with the stretched exponential model: $I=I_{\mathrm{o}} \exp \left[-(t / \tau)^{\beta}\right]$, which yields values of the stretching parameter $\beta=0.84 \pm 0.06$ and a recombination lifetime of $\tau=711 \mathrm{ps}$. At the measurement temperature of $10 \mathrm{~K}$, the latter represents the radiative lifetime of the biexciton emission, which is significantly lower than that measured in comparable $\mathrm{In}_{0.25} \mathrm{Ga}_{0.75} \mathrm{~N}$ quantum wells ${ }^{46}$. This could be attributed to a relatively strong three-dimensional confinement that leads to a strong electron-hole overlap in the QD. The short radiative lifetime suggests the suppression of polarization field in the dotin-nanowire heterostructure, as previously indicated by the invariance of the peak energy with excitation. It may also be noted that the QD biexciton decay lifetime measured here is 

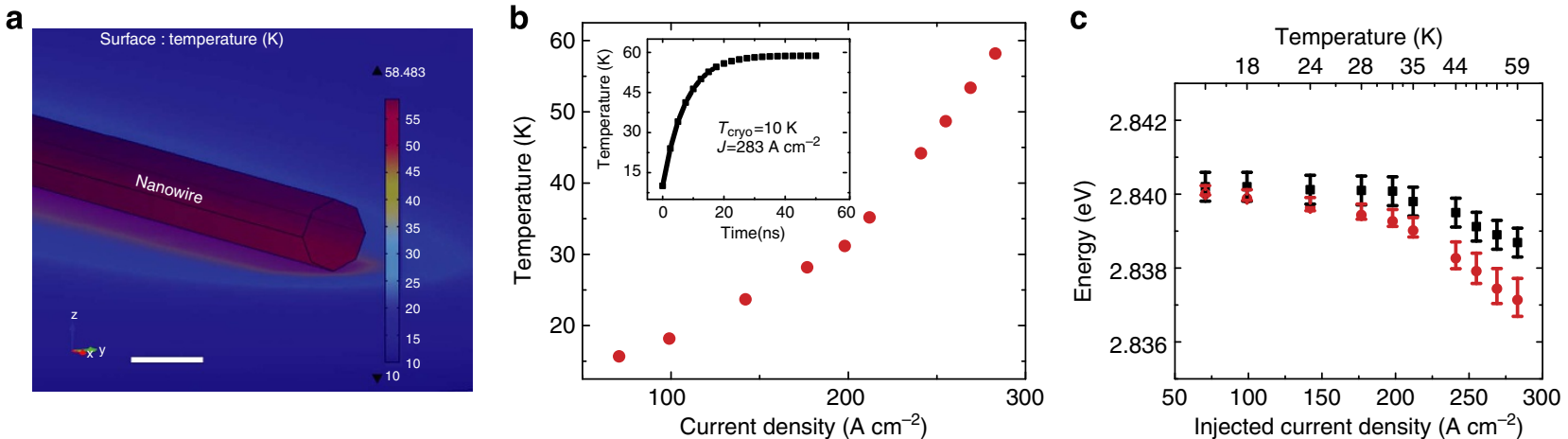

Figure 4 | Single dot-in-nanowire p-n junction diode temperature. (a) Steady-state spatial temperature profile of a 600-nm-long nanowire of $30 \mathrm{~nm}$ diameter placed on a Si/ $\mathrm{SiO}_{2}$ platform for an injection current density of $283 \mathrm{~A} \mathrm{~cm}{ }^{-2}$. The scale bar denotes $30 \mathrm{~nm}$. The silicon substrate is maintained at $10 \mathrm{~K}$ and the final nanowire temperature is $\sim 59 \mathrm{~K}$. (b) Calculated device temperature for different values of current density. The inset shows the transient response of the nanowire temperature for an injected current density of $283 \mathrm{~A} \mathrm{~cm}^{-2}$. The transient response depends on the thermal resistance and capacitance of the system. (c) Emission energy shift as a function of the injected current density. The dots represent energy values calculated with the Varshni equation for equivalent temperatures corresponding to the current densities, obtained from the data shown in $\mathbf{b}$. The error bars represent uncertainties in the Varshni fitting parameters. The squares represent measured variation of the exciton emission peak energy with injection current. Errors in the measurement arise from the resolution of the monochromator.

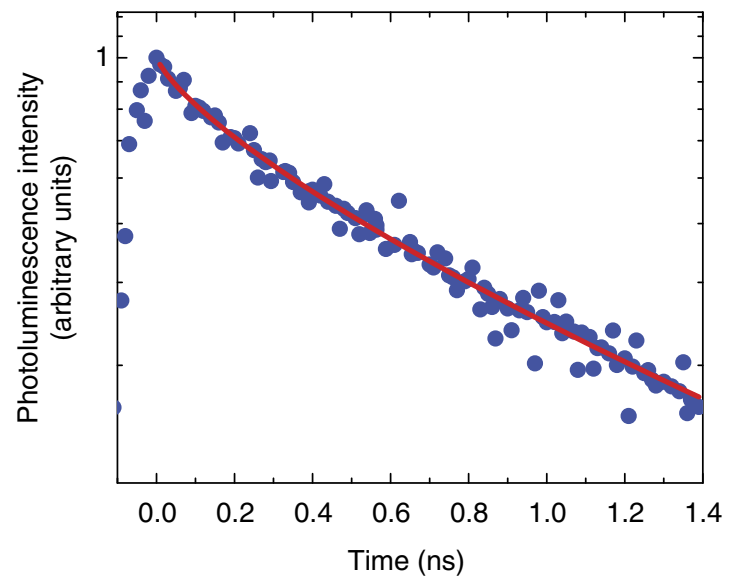

Figure 5 | Temporal variation of luminescence intensity from a single $\mathbf{I n}_{\mathbf{0 . 2 5}} \mathbf{G a}_{0.75} \mathbf{N}$ dot-in-nanowire measured at the biexciton energy. The luminescence is detected at $10 \mathrm{~K}$, by a single-photon avalanche photodiode with a response time of $40 \mathrm{ps}$. The solid curve indicates values calculated with the stretched exponential model. The fitting yields lifetime $\tau=711 \mathrm{ps}$ and $\beta=0.84 \pm 0.06$. The adjusted $R^{2}$ value for the fit is 0.96 .

significantly smaller than decay times reported for other nitride QD-based single-photon emitters ${ }^{6,12}$. The QD-nanowire diode is therefore promising as a single-photon source operating at high frequencies.

Photon correlation statistics. For an ideal single-photon source, the second-order correlation function: $g^{(2)}(\tau)=\langle I(t) I(t+\tau)>$ / $<I(t)>\langle I(t+\tau)>$, where $\tau$ is the time delay and $I(t)$ is the electroluminescence intensity at time $t$, should be equal to zero at zero time delay. Figure $6 \mathrm{a}, \mathrm{b}$ exhibit the second-order correlation measured in a standard Hanbury Brown and Twiss experiment for the exciton and biexciton resonances, respectively. The second-order correlation was measured under continuous excitation below saturation, at an injection current density of $\sim 142 \mathrm{~A} \mathrm{~cm}^{-2}$ $(I=1 \mathrm{nA})$. The comparatively large separation between exciton and biexciton lines and narrow emission linewidths at $10 \mathrm{~K}$ allow us to spectrally filter and study each transition as a single-photon emitter. The dip in the correlation spectrum at zero time delay indicates anti-bunching in both exciton and biexciton complexes. We have analysed the correlation data for both peaks with the function: $g^{(2)}(\tau)=1-a \times \exp (-\tau / b)$ where $(1-a)$ and $b$ are the $g^{(2)}(0)$ and recombination lifetime respectively. The solid lines represent the calculated values of $g^{(2)}(\tau)$. The value of $g^{(2)}(0)$ for the exciton transition is 0.3 , which corresponds to a 3 -fold decrease in unwanted multi-photon emission events compared with attenuated coherent light sources. The anti-bunching dip is shallower in case of the biexciton emission, where $g^{(2)}(0)=0.38$. This may be due to the faster recovery of $g^{(2)}(\tau)$ of the biexciton with a shorter lifetime, compared with that of the exciton. The analysis gives exciton and biexciton emission lifetimes of 1.1 and $0.7 \mathrm{~ns}$, respectively. The latter value is in excellent agreement with that obtained from the TRPL measurement. The finite value of the second-order correlation at zero time delay may be attributed to the finite time resolution of the measurement system and the background. To eliminate the effect of background counts, which could originate from stray light or merely dark counts, we apply a correction: $g^{(2)}(0)=1+\left(g^{(2)}\right.$ meas $\left.(0)-1\right) / \rho^{2}$, where $\rho$ is the ratio of signal-to-total counts, including dark counts ${ }^{47}$. The $g^{(2)}(0)$ values derived after background correction for the exciton and biexciton emission are 0.16 and 0.25 , respectively.

Output polarization. The polarization of the emitted single photons was also investigated by placing a linear polarizer in front of the monochromator. The measured intensity of the single dot biexciton emission as a function of the angle of the polarizer from the $c$ axis of the nanowire is shown in the polar plot of Fig. 6c. The solid line indicates the fit of the experimental data to the function: $I(\theta)=a+b \cos ^{2}\left(\theta-\theta_{0}\right)$ where $a$ and $(a+b)$ are minimum and maximum values of the PL intensity, respectively, $\theta$ is the polarizer angle and $\theta_{0}$ is the angle of polarization. The $\mathrm{QD}$ emission is linearly polarized with a degree of polarization, $\rho=\left(I_{\|}-I_{\perp}\right) /\left(I_{\|}+I_{\perp}\right)$ of $\sim 70 \%$ along the $c$ axis of the nanowire, where $I_{\|}\left(I_{\perp}\right)$ denotes the light intensity parallel (perpendicular) to the $c$ axis of the nanowire. Strong linear polarization along the nanowire is attributed to the large dielectric constant contrast between the nanowire and the surrounding air, attenuating the electric field amplitude in the direction perpendicular to the nanowire ${ }^{48}$. 
a

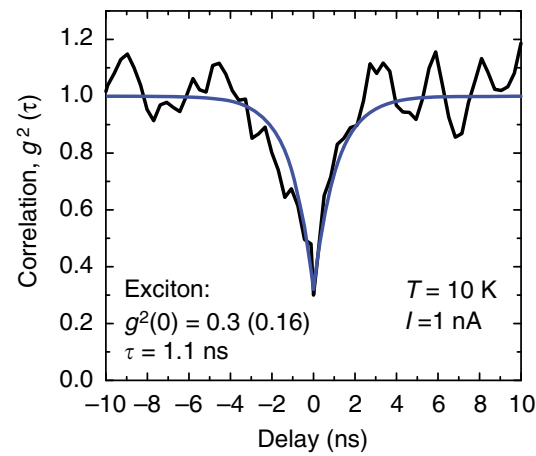

b

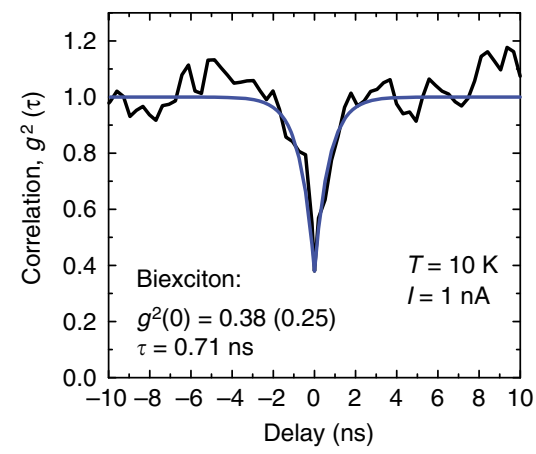

c

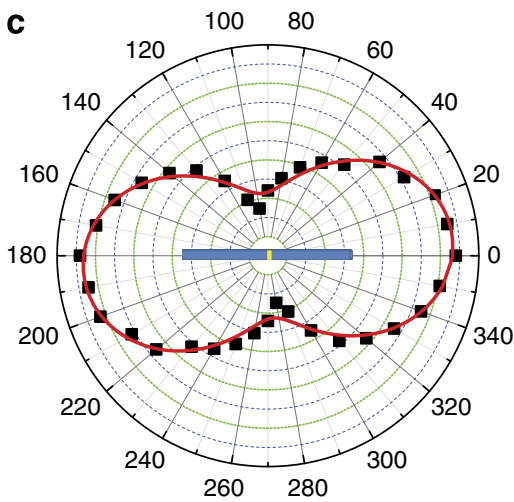

Figure 6 | Electrically injected single-photon emission from InGaN quantum dot-in-nanowire. Second-order correlation measured at $10 \mathrm{~K}$ with continuous electrical injection at (a) Exciton energy and (b) Biexciton energy. The solid lines are fits to the data as explained in the text. (c) Polar plot of electroluminescence intensity as a function of polarizer angle. The nanowire orientation is schematically superimposed.

\section{Discussion}

It should be noted that the red shift in the QD emission due to increase of temperature associated with Joule heating is smaller than the value theoretically predicted by the Varshni equation. We attribute this difference to a small blue shift $(<2 \mathrm{meV})$ that is likely to arise from the screening of the very small polarization field with excitation. As a consequence of the two competing effects, we observe that the excitonic emission remains almost invariant with injection. Typically, nitride-based quantum wells and self-organized QDs show blue shifts of the order of tens of meVs due to screening of the piezoelectric field and band filling of localized states ${ }^{49,50}$. The negligibly small blue shift in our device indicates that both these effects are greatly suppressed due to the dot-in-nanowire geometry. Pseudomorphic strain in InGaN/GaN and GaN/AlGaN nanowires has been known to relax efficiently at the sidewalls during growth ${ }^{37,51,52}$. Consequently, the piezoelectric field in such dot-in-nanowire heterostructures is significantly lower than that in quantum wells or self-organized QDs. Additionally, band filling effects induced by compositional inhomogeneities and alloy disorders, which lead to excitation dependent energy shifts, are also absent in this device.

At $10 \mathrm{~K}$, the biexciton decay time $\tau=\tau_{\text {rad }}=0.71 \mathrm{~ns}$. The biexciton lifetime derived from analysis of the second-order correlation data is also $0.7 \mathrm{~ns}$. The second-order correlation was measured at an injection current density of $142 \mathrm{~A} \mathrm{~cm}^{-2}$, for which we estimate the actual device temperature to be $\sim 25 \mathrm{~K}$ due to Joule heating. The close agreement between the two lifetime values indicates that the electrically injected carriers do not encounter recombination through non-radiative channels and both lifetime values correspond almost exclusively to radiative recombination. From temperature-dependent lifetime measurements performed on similar dot-in-nanowire ensemble samples, we have observed that the radiative lifetime is nearly constant with temperature. Hence, it can be assumed that the biexciton decay rate in our single dot device would be $\sim 0.7 \mathrm{~ns}$ at higher temperatures. The relatively small radiative decay time is a remarkable advantage of the dot-in-nanowire geometry as it potentially allows for large repetition rates in single-photon emitters. Finally, the nanowires were grown on (111) Si and the single nanowire junction devices were fabricated on (001) $\mathrm{Si}$, which make the single-photon sources reported here very attractive from the standpoint of compatibility and integration with silicon technology. However, operation of the device at higher temperatures will require engineering of the dot heterostructure to allow for better carrier confinement in the QD. Further optimization of the ohmic contact technology will be important to reduce the series resistance of the device. Due to growth-related inhomogeneities in the InGaN disk in the nanowires, the characteristics varied by a small degree from device to device. The results presented here represent the best ones measured.

\section{Methods}

Epitaxial growth of nanowires on silicon. The InGaN/GaN dot-in-nanowire heterostructure, shown schematically, was grown on (111) Si substrate by PA-MBE. After standard cleaning with solvents and removal of surface oxides with a dilute hydrofluoric acid solution, the substrate was annealed in the MBE chamber at $900^{\circ} \mathrm{C}$ for $60 \mathrm{~min}$ before growth to remove any residual native oxide. Conventionally, the growth of GaN nanowires is initiated by depositing a few monolayers of Ga that act as a catalyst for subsequent growth of the nanowires. However, we found that under optimum growth conditions defect-free $\mathrm{GaN}$ nanowires can be grown on $\mathrm{Si}$ without the initial self-catalysis step. A striking difference in the nanowires grown by the two techniques lies in their diameters. It was observed that nanowires grown without the seed $\mathrm{Ga}$ deposition have an average diameters of $\leq 25 \mathrm{~nm}$. Growth of the nanowire samples was carried out in a nitrogen-rich environment, while maintaining a constant $\mathrm{N}_{2}$ flux of 1.0 s.c.c.m. The growth temperature was maintained at $800^{\circ} \mathrm{C}$ and the $\mathrm{Ga}$ flux at $10^{-7}$ Torr. Three hundred nanometre of Si-doped $\mathrm{GaN}\left(n \sim 3 \times 10^{18} \mathrm{~cm}^{-3}\right)$ was first grown, followed by the active region consisting of a $2-\mathrm{nm} \mathrm{In}_{0.25} \mathrm{Ga}_{0.75} \mathrm{~N}$ QD at the centre of $30 \mathrm{~nm}$ of undoped GaN. During the growth of the QD, the substrate temperature was lowered to $535^{\circ} \mathrm{C}$ and an in flux of $6 \times 10^{-8}$ Torr was used. Subsequently, $250 \mathrm{~nm}$ of $\mathrm{Mg}$-doped p-type $\mathrm{GaN}\left(p \sim 5 \times 10^{17} \mathrm{~cm}^{-3}\right)$ was grown at $720^{\circ} \mathrm{C}$ to allow for efficient $\mathrm{Mg}$ incorporation. The $\mathrm{Mg}$ flux was increased during the growth of the final $20 \mathrm{~nm}$ of $\mathrm{p}-\mathrm{GaN}$ to achieve a better ohmic contact to a single nanowire. Lowering of the substrate temperature during the growth of $\mathrm{p}-\mathrm{GaN}$ leads to an increase in the lateral growth rate and results in an increase in the nanowire diameter at the $\mathrm{p}-\mathrm{GaN}$ end.

TEM imaging. Nanowires from the as-grown sample were mechanically removed from the substrate and ultrasonicated in isopropyl alcohol (IPA) for $20 \mathrm{~min}$. Few drops of the nanowire IPA solution were then put on a carbon-coated TEM grid. After evaporation of the liquid, the nanowires on the grid were imaged using a JEOL 3011 high-resolution TEM.

QD composition analysis. STEM imaging and EDX measurements were performed on a single nanowire on a Cs-corrected JEOL 2100F TEM at an accelerating voltage of $200 \mathrm{kV}$. Before imaging and spectroscopy, the nanowires were dispersed on a holey carbon grid. A Hitachi SU8000 SEM with EDX capability was used to calibrate the Indium composition in InGaN nanowires, grown under identical conditions.

Device fabrication. The nanowires were dispersed on a (001) silicon wafer covered with $100 \mathrm{~nm}$ of thermal $\mathrm{SiO}_{2}$. Single nanowires were isolated by SEM imaging and $\mathrm{Ti} / \mathrm{Au}$ ohmic contacts $(5 \mathrm{~nm} / 45 \mathrm{~nm})$ with large contact pads were defined at the two ends of a single nanowire, $\sim 600 \mathrm{~nm}$ in length, using electron beam lithography and electron beam evaporation techniques. The contacts were annealed in a $\mathrm{N}_{2}$ ambient at $450^{\circ} \mathrm{C}$ for $30 \mathrm{~min}$ and the devices were wire bonded onto chip carriers for measurements. 
Single dot spectroscopy and time-resolved PL. Single QD spectroscopy was performed with a micro-PL measurement system in the far field in a confocal geometry. The sample was mounted on the cold finger of a continuous flow helium cryostat. Luminescence collected by an objective lens $(\times 50)$ in the normal direction was analysed with a high-resolution monochromator with a spectral resolution of $\sim 0.03 \mathrm{~nm}$ and detected with a photomultiplier tube using phasesensitive lock-in amplification. For PL measurements, the same microscope objective was used to focus light from a frequency tripled Ti:sapphire laser $(\lambda=267 \mathrm{~nm}$, repetition rate $=80 \mathrm{MHz})$ on to the sample and to collect the luminescence from the InGaN/GaN QD. The photoexcitation spot size was $\sim 5 \mu \mathrm{m}$ and a pin hole was used to spatially resolve a single QD. For electroluminescence measurements, a continuous current is injected into the single nanowire device using a Keithley 6200 precision current source. For time-resolved PL measurements, the biexcitonic peak was filtered using a high-resolution monochromator and detected using a Picoquant single-photon avalanche photodiode with a response time of $40 \mathrm{ps}$.

Second-order correlation measurements. The second-order correlation of the exciton and biexciton was measured under continuous electrical excitation using a Hanbury Brown and Twiss measurement system. The exciton or biexciton luminescence was filtered from the QD emission and transmitted through a 50:50 beam splitter and on to two single-photon detectors, which provide the start and stop pulses to the counter.

Temperature simulation. The temperature of the nanowire was estimated by solving the Laplace equation for heat transfer using COMSOL Multiphysics software. The device is modelled as a uniform nanowire lying on $100 \mathrm{~nm}$ of thermal $\mathrm{SiO}_{2}$ on the surface of a $500-\mu \mathrm{m}$ silicon wafer, which is cooled to $10 \mathrm{~K}$ by the cold finger of the cryostat. The GaN region of the nanowire is the Joule heat source and the silicon wafer is effectively an infinite heat sink. The thermal oxide presents a thermal resistance between the nanowire and the heat sink. Heat loss due to convection may be ignored as the cryostat is evacuated before it is cooled down to $10 \mathrm{~K}$. Radiation effects are also negligible as the temperature and area under consideration are small. Low temperature values of all material constants were considered.

\section{References}

1. Michler, P. et al. A quantum dot single-photon turnstile device. Science 290, 2282-2285 (2000)

2. Michler, P. et al. Quantum correlation among photons from a single quantum dot at room temperature. Nature 406, 968-970 (2000).

3. Santori, C., Pelton, M., Solomon, G., Dale, Y. \& Yamamoto, Y. Triggered single photons from a quantum dot. Phys. Rev. Lett. 86, 1502-1505 (2001).

4. Zwiller, V. et al. Single quantum dots emit single photons at a time: antibunching experiments. Appl. Phys. Lett. 78, 2476-2478 (2001).

5. Sebald, K. et al. Single-photon emission of CdSe quantum dots at temperatures up to 200 K. Appl. Phys. Lett. 81, 2920-2922 (2002).

6. Kako, S. et al. A gallium nitride single-photon source operating at $200 \mathrm{~K}$. Nature Mater. 5, 887-892 (2006).

7. Fedorych, O. et al. Room temperature single photon emission from an epitaxially grown quantum dot. Appl. Phys. Lett. 100, 061114 (2012)

8. Reimer, M. et al. Bright single-photon sources in bottom-up tailored nanowires. Nat. Commun. 3, 737 (2012).

9. Yuan, Z. et al. Electrically driven single-photon source. Science 295, 102-105 (2002).

10. Bhattacharya, P., Das, A., Basu, D., Guo, W. \& Heo, J. An electrically injected quantum dot spin polarized single photon source. Appl. Phys. Lett. 96, 101105 (2010).

11. Heindel, T. et al. Electrically driven quantum dot-micropillar single photon source with 34\% overall efficiency. Appl. Phys. Lett. 96, 011107 (2010).

12. Jarjour, A. et al. Cavity-enhanced blue single-photon emission from a single InGaN/GaN quantum dot. Appl. Phys. Lett. 91, 052101 (2007).

13. Kremling, S. et al. Single photon emission from InGaN/GaN quantum dots up to 50 K. Appl. Phys. Lett. 100, 061115 (2012).

14. Bounouar, S. et al. Ultrafast room temperature single-photon source from nanowire-quantum dots. Nano Lett. 12, 2977-2981 (2012).

15. Strauf, S. et al. High-frequency single-photon source with polarization control. Nat. Photon. 1, 704-708 (2007).

16. Claudon, J. et al. A highly efficient single-photon source based on a quantum dot in a photonic nanowire. Nat. Photon. 4, 174-177 (2010).

17. Thompson, T. M. et al. Single-photon emission from exciton complexes in individual quantum dots. Phys. Rev. B 64, 201302 (2001).

18. Shields, A. J. Semiconductor quantum light sources. Nat. Photon. 1, 215-223 (2007).

19. Arians, R. et al. Electrically driven single quantum dot emitter operating at room temperature. Appl. Phys. Lett. 93, 173506 (2008).
20. Robert, I., Moreau, E., Gérard, J. \& Abram, I. Towards a single-mode single photon source based on single quantum dots. J. Lumin. 94-95, 797-803 (2001).

21. Walls, D. F. \& Milburn, G. J. Quantum Optics (Springer, 1994).

22. Muller, A., Breguet, J. \& Gisin, N. Experimental demonstration of quantum cryptography using polarized photons in optical fibre over more than $1 \mathrm{~km}$. Europhys. Lett. 23, 383-388 (1993).

23. Bertness, K., Sanford, A. \& Davydov, A. GaN nanowires grown by molecular beam epitaxy. IEEE J. Select. Topics Quantum Electron 17, 847-858 (2011).

24. Cerutti, L. et al. Wurtzite $\mathrm{GaN}$ nanocolumns grown on $\mathrm{Si}(001)$ by molecular beam epitaxy. Appl. Phys. Lett. 88, 213114 (2006).

25. Kishino, K., Kikuchi, A., Sekiguchi, H. \& Ishizawa, S. InGaN/GaN nanocolumn LEDs emitting from blue to red. Proc. SPIE 6473, 64730T (2007).

26. Calarco, R. et al. Nucleation and growth of $\mathrm{GaN}$ nanowires on $\mathrm{Si}(111)$ performed by molecular beam epitaxy. Nano Lett. 7, 2248-2251 (2007).

27. Bertness, K., Roshko, A., Mansfield, L., Harvey, T. \& Sanford, N. Nucleation conditions for catalyst-free GaN nanowires. J. Cryst. Growth 310, 3154-3158 (2008).

28. Fernandez-Garrido, S., Grandal, J., Calleja, E., Sanchez-Garcia, M. \& LopezRomero, D. A growth diagram for plasma-assisted molecular beam epitaxy of GaN nanocolumns on Si(111). J. Appl. Phys. 106, 126102 (2009).

29. Landré, O., Bougerol, C., Renevier, H. \& Daudin, B. Nucleation mechanism of $\mathrm{GaN}$ nanocolumns grown on (111) Si by molecular beam epitaxy. Nanotechnology 20, 415602 (2009).

30. Consonni, V., Knelangen, M., Geelhaar, L., Trampert, A. \& Riechert, H. Nucleation mechanisms of epitaxial GaN nanowires: origin of their selfinduced formation and initial radius. Phys. Rev. B 81, 085310 (2010).

31. Guo, W., Zhang, M., Banerjee, A. \& Bhattacharya, P. Catalyst-free InGaN/GaN nanowire light emitting diodes grown on (001) silicon by molecular beam epitaxy. Nano Lett. 10, 3355-3359 (2010).

32. Armstrong, A. et al. Deep level optical spectroscopy of GaN nanorods. J. Appl Phys. 106, 053712 (2009).

33. Duan, X. \& Lieber, C. Laser-assisted catalytic growth of single crystal GaN nanowires. C. J. Am. Chem. Soc. 122, 188-189 (2011).

34. Kuykendall, T., Ulrich, P., Aloni, S. \& Yang, P. Complete composition tunability of InGaN nanowires using a combinatorial approach. Nat. Mater. 6 , 951-956 (2007).

35. Sanford, N. et al. Steady-state and transient photoconductivity in $c$-axis GaN nanowires grown by nitrogen-plasma-assisted molecular beam epitaxy. J. Appl. Phys. 107, 034318 (2010).

36. Ristić, J. et al. Characterization of GaN quantum discs embedded in $\mathrm{Al}_{x} \mathrm{Ga}_{1-x} \mathrm{~N}$ nanocolumns grown by molecular beam epitaxy. Phys. Rev. B 68, 125305 (2003).

37. Renard, J., Songmuang, R., Bougerol, C., Daudin, B. \& Gayral, B. Exciton and biexciton luminescence from single GaN/AlN quantum dots in nanowires. Nano Lett. 8, 2092-2096 (2008).

38. Guo, W., Banerjee, A., Bhattacharya, P. \& Ooi, B. InGaN/GaN disk-in-nanowire white light emitting diodes on (001) silicon. Appl. Phys. Lett. 98, 193102 (2011).

39. Tan, L., Martin, R., O’Donnell, K. \& Watson, I. Photoluminescence and phonon satellites of single InGaN/GaN quantum wells with varying $\mathrm{GaN}$ cap thickness. Appl. Phys. Lett. 89, 101910 (2006).

40. Chen, X. et al. Straight and smooth GaN nanowires. Adv. Mater. 12, 1432-1434 (2000).

41. Varshni, Y. Temperature dependence of the energy gap in semiconductors. Physica 39, 149-154 (1967).

42. Nepal, N., Li, J., Nakarmi, M., Lin, J. \& Jiang, H. Exciton localization in AlGaN alloys. Appl. Phys. Lett. 88, 062103 (2006).

43. Steude, G. et al. Large-scale synthesis of single crystalline gallium nitride nanowires. Appl. Phys. Lett. 74, 2456-2458 (1999).

44. Ferguson, I., Cheng, T., Torres, C. \& Murray, R. Photoluminescence of molecular beam epitaxial grown $\mathrm{Al}_{0.48} \mathrm{In}_{0.52}$ As. J. Vac. Sci. Technol. B 12, 1319-1327 (1994).

45. Schömig, H. et al. Probing individual localization centers in an InGaN/GaN quantum well. Phys. Rev. Lett. 92, 106802 (2004)

46. Chen, C., Lu, Y., Yeh, D. \& Yang, C. Influence of the quantum-confined Stark effect in an InGaN/GaN quantum well on its coupling with surface plasmon for light emission enhancement. Appl. Phys. Lett. 90, 183114 (2007).

47. Brouri, R., Beveratos, A., Poizat, J. \& Grangier, P. Photon antibunching in the fluorescence of individual color centers in diamond. Opt. Lett. 25, 1294-1296 (2000).

48. Wang, J., Gudiksen, M., Duan, X., Cui, Y. \& Lieber, C. Highly polarized photoluminescence and photodetection from single indium phosphide nanowires. Science 293, 1455-1457 (2001).

49. Chichibu, S., Azuhata, T., Sota, T. \& Nakamura, S. Spontaneous emission of localized excitons in InGaN single and multiquantum well structures. Appl. Phys. Lett. 69, 4188-4190 (1996).

50. Jho, Y., Yahng, J., Oh, E. \& Kim, K. Field-dependent carrier decay dynamics in strained $\operatorname{In}_{x} \mathrm{Ga}_{1-x} \mathrm{~N} / \mathrm{GaN}$ quantum wells. Phys. Rev. B 66, 035334 (2002). 
51. Guo, W., Zhang, M., Bhattacharya, P. \& Heo, J. Auger Recombination in III-nitride nanowires and its effect on nanowire light-emitting diode characteristics. Nano Lett. 11, 1434-1438 (2011).

52. Kawakami, Y. et al. Origin of high oscillator strength in green-emitting InGaN/ GaN nanocolumns. Appl. Phys. Lett. 89, 163124 (2006).

\section{Acknowledgements}

The work is supported by the National Science Foundation (MRSEC programme) under Grant DMR-1120923. Device fabrication was done in the Lurie Nanofabrication Facility at the University of Michigan, a member of the National Nanotechnology Infrastructure Network funded by the National Science Foundation. We thank Dr K. Sun for valuable help with STEM and EDX measurements.

\section{Author contributions}

All authors contributed to the design of this work and preparation of the manuscript. S.D. performed the growth, TEM, SEM characterization and device temperature simulation. S.D. and J.H. performed the device fabrication. S.D. and A.D. performed the luminescence measurements. All authors discussed the results and implications and commented on the manuscript at all stages.

\section{Additional information}

Supplementary Information accompanies this paper at http://www.nature.com/ naturecommunications

Competing financial interests: The authors declare no competing financial interests.

Reprints and permission information is available online at http://npg.nature.com/ reprintsandpermissions/

How to cite this article: Deshpande, S. et al. Electrically driven polarized single-photon emission from an InGaN quantum dot in a GaN nanowire. Nat. Commun. 4:1675 doi: $10.1038 /$ ncomms2691 (2013). 